\title{
Task-based working memory guidance of visual attention
}

\author{
Zhe Chen • Brian H. Tsou
}

Published online: 20 January 2011

(C) Psychonomic Society, Inc. 2011

\begin{abstract}
Previous research has established that holding a stimulus in working memory (WM) facilitates the deployment of visual attention to that stimulus relative to other stimuli. The present study examined whether maintaining a specific task in WM would also bias the allocation of attention to the stimuli associated with that task. Participants performed a speeded letter search task while simultaneously keeping in WM one of two task cues shown at the beginning of each trial. The results showed that taskbased WM guidance of attention was modulated by response latencies. Whereas the participants with fast reaction times showed little influence of WM contents, the participants with slow reaction times took longer to respond when the letter target appeared in a distractor stimulus consistent with the task cue held in mind. A subsequent Stroop experiment found a larger Stroop interference effect from the participants in the slow group compared with those in the fast group, suggesting that the differential WM effect between the two groups may be associated with an individual's ability to inhibit taskirrelevant information. Taken together, these results expanded the realm of previous research and provided further evidence for a close link between attention and WM.
\end{abstract}

Keywords Working memory - Attention · Visual search . Distractor inhibition

\footnotetext{
Z. Chen $(\triangle)$

Department of Psychology, University of Canterbury,

Private Bag 4800,

Christchurch, New Zealand

e-mail: zhe.chen@canterbury.ac.nz

B. H. Tsou

U.S. Air Force Research Laboratory,

Wright-Patterson Air Force Base, OH, USA
}

Visual perception is inherently selective (Palmer, 1999). One way to understand this selectivity is to understand the mechanisms that control the deployment of attention. Broadly speaking, there are two types of processes that control attention: stimulus-driven (or bottom-up) processes that capture attention by specific stimulus properties independent of task relevancy, and goal-directed (or topdown) processes that guide the deployment of attention consistent with one's behavioral goals (see Egeth \& Yantis, 1997, for a review). One form of top-down control is the guidance of visual attention by the contents of working memory (see, e.g., Downing, 2000; Huang \& Pashler, 2007; Moores, Latti, \& Chelazzi, 2003; Olivers, Meijer, \& Theeuwes, 2006; Pashler \& Shui, 1999; Soto, Heinke, Humphreys, \& Blanco, 2005). Simply put, objects that match the currently active mental contents are preferentially attended to. The present study focuses on this topic.

Many studies have reported a close link between working memory (WM) and visual attention. There is considerable evidence that paying attention to a stimulus or maintaining the stimulus in WM facilitates the processing of that stimulus. For example, attended objects tend to be remembered better than unattended objects (e.g., Bundesen, 1990; Reeves \& Sperling, 1986; Smyth, 1996). Furthermore, when a location is held in WM, the processing of stimuli at that location is facilitated relative to the processing of stimuli at other locations (e.g., Awh \& Jonides, 2001). Single-neuron recordings and neuroimaging studies have also revealed a large overlap in the brain areas that are involved in both visual attention and WM (for reviews, see Awh \& Jonides, 2001; Cabeza \& Nyberg, 2000). It has been shown that attending to an object enhances the neuronal responses in many visual areas of the brain, including the early visual areas, and the inferotemporal, parietal, and prefrontal cortices (e.g., Chelazzi, Miller, Duncan, \& Desimone, 1993; Kastner, De Weerd, 
Desimone, \& Ungerleider, 1998; Miller, Erickson, \& Desimone, 1996; Moran \& Desimone, 1985; Rainer, Asaad, \& Miller, 1998; Roelfsema, Lamme, \& Spekreijse, 1998; Wojciulik \& Kanwisher, 1999). In addition, holding some aspects of an object in WM without seeing the object directly increases the firing rate of neurons in many of these same areas (e.g., Chelazzi et al., 1993; Gnadt \& Andersen, 1988; Miller et al., 1996; Rainer et al., 1998; Supèr, Spekreijse, \& Lamme, 2001).

WM has also been found to affect the deployment of visual attention (e.g., Downing, 2000; Huang \& Pashler, 2007; Moores et al., 2003; Olivers et al., 2006; Pashler \& Shui, 1999; Soto et al., 2005). In a typical experiment, participants first remember a stimulus (the memory sample), then do a selective attention task (e.g., search for a target among distractors or make a binary discrimination judgment), and finally perform a memory test. Importantly, the memory sample does not provide information about the target in the selective attention task. Thus, if attention is not influenced by what is actively held in WM, then the location of the memory sample, or its presence or absence in a search display, should have no effect on the response latencies of the target in the attention task. However, contrary to the above prediction, Downing showed that his participants were faster to respond to a probe that appeared at a location previously occupied by a memory sample relative to a stimulus not held in WM. Similar results were found by Huang and Pashler, whose participants reported a digit more often when it appeared on an object that matched the memory sample than on a different object. These results suggest that the contents of WM facilitated the deployment of visual attention.

The contents of WM can also delay responses in visual search tasks, or they can have no effects on the deployment of attention under some circumstances. These results typically occur when participants know in advance that the memory sample, if present, can only serve as distractors in a visual search display (e.g., Downing \& Dodds, 2004; Olivers et al., 2006; Soto et al., 2005; Woodman \& Luck, 2007). For example, Olivers et al. (2006) showed that their participants took longer to perform a letter task when a search display contained a singleton distractor that matched rather than mismatched the memory sample. Downing and Dodds also found no evidence of attentional capture by WM when the memory sample could only appear as a distractor in a search display. Han and Kim (2009) divided their participants into two groups based on the participants' reaction times (RTs) in a neutral condition (i.e., the search display did not contain the memory sample) and found that the effect of WM was modulated by the participants' response latencies. Whereas the WM effect was enhanced for the fast group, it was reduced for the slow group. In subsequent experiments, Han and Kim manipulated the duration of the foreknowledge of the search target and found evidence for WM capture when the duration was short, but not when it was long. These results suggest that the effect of WM was influenced by the level of cognitive control that could be exerted over the contents of WM.

A common feature in prior research has been that the participants were required to hold an episodic representation of a specific stimulus in WM. Typically, they saw one of several memory samples on each trial. In order to perform the memory task at the end of a trial, they needed to maintain the representation of a given memory sample. In everyday life, however, although we often keep specific items in mind, we also hold specific tasks in WM. We may be thinking, for instance, of getting some fruit on the way home without any specific type of fruit in mind. In other words, WM can be task-oriented (e.g., thinking of buying fruit rather than buying meat) in addition to being stimulus-oriented. The question is: does holding a task in WM also influence the deployment of visual attention?

The present study examined the effect of task-based WM on attention. As in prior research, each trial consisted of a memory display, followed by a visual search display where the critical manipulation occurred between the contents of WM and a target that required a speeded response, and finally, by an object display that required participants to use the information they held in WM (see Fig. 1b). Importantly, the present experiments differed from the previous experiments in two ways. First, the memory display consisted of a verbal task cue (the word "Shape" or "Gap") instead of a specific memory sample. This was to emphasize the importance of a specific task rather than of a specific stimulus. Second, there was no explicit memory test at the end of a trial. Instead, memory for the task cue was assessed via an object identification task. At the end of each trial, participants saw two objects, one belonging to the "shape" task and the other to the "gap" task, and which object they responded to was based on the task cue. The use of an object identification task instead of an explicit recognition memory task was to eliminate what Woodman and Luck (2007) referred to as "strategic perceptual resampling" - that is, deploying attention to the object that matched the memory sample to refresh the memory of that object. When an experiment uses an explicit memory test, so long as attending to the memory sample can facilitate performance in the memory test, it is virtually impossible to ensure that participants do not engage in strategic perceptual resampling while the search display is on. In the present study, the stimulus consistent with the task cue, if present, had nothing to do with the correct responses in either the selective attention or the object identification task. It was hoped that this design feature would discourage 
Fig. 1 Examples of stimulus displays from Experiment 1. In both Fig. 1a and b, the hexagons/octagons were red, and the rectangles with a gap at the bottom were green. All the other stimuli were black. (a) An example of a display from the shape/gap practice block. (b) An example of a display from the experimental block. SC refers to the singleton-consistent condition, where the target was in a singleton consistent with the task cue. SI refers to the singleton-inconsistent condition, where the target was in a singleton inconsistent with the task cue. NC refers to the neutralconsistent condition, where the target was in a neutral object, and the singleton in the display was consistent with the task cue. NI refers to the neutralinconsistent condition, where the target was in a neutral object, and the singleton in the display was inconsistent with the task cue
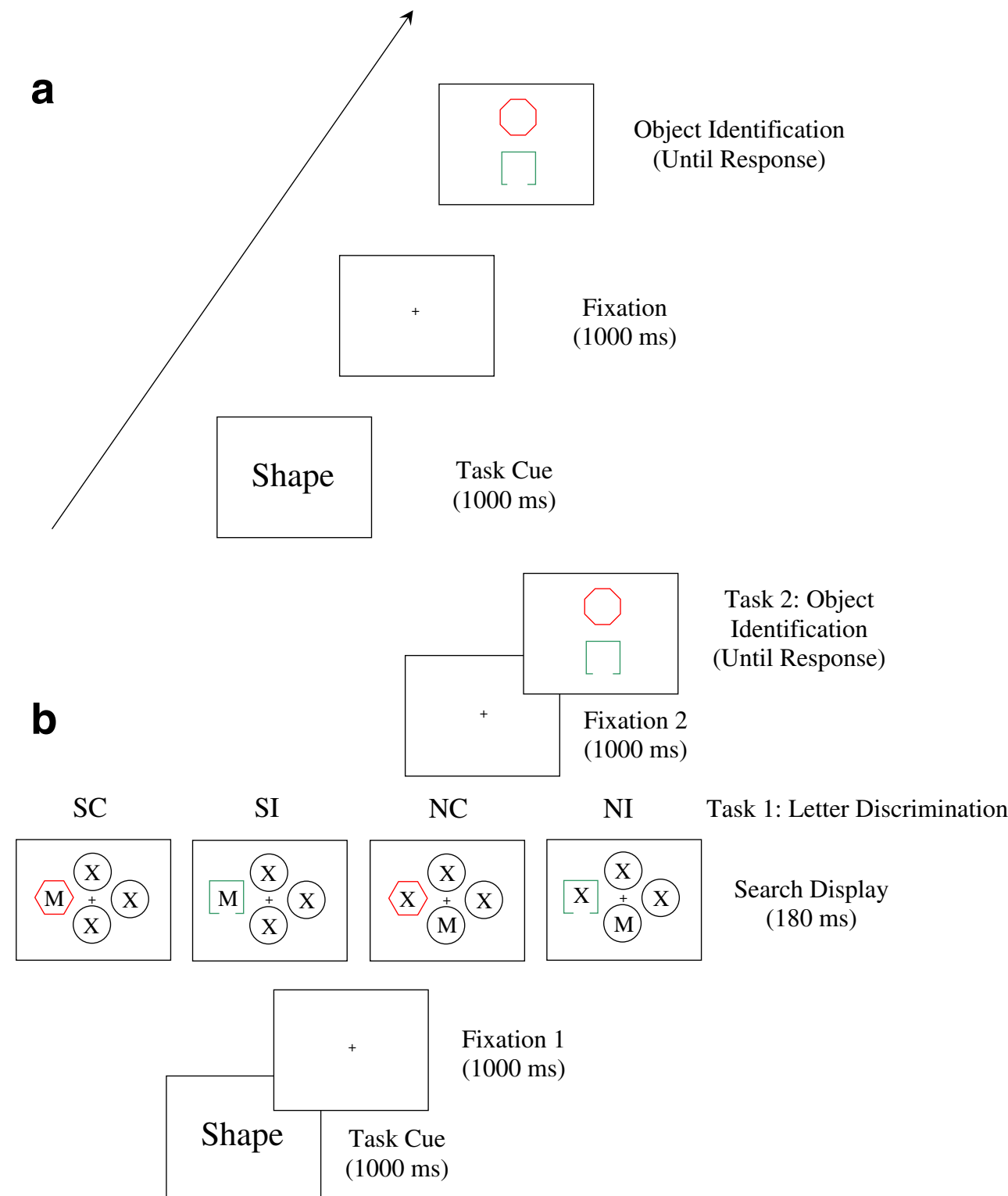

participants from engaging in strategic perceptual resampling, thereby preventing biased results.

\section{Experiment 1}

Experiment 1 investigated the relationship between taskbased WM contents and the deployment of visual attention. Participants performed two tasks on each trial: a speeded letter search task and an accuracy-only object identification task. The target in the letter search task could appear in either a singleton or one of three nonsingleton, neutral objects, and the singleton was equally likely to be consistent or inconsistent with the task cue. Since singletons are known to capture attention in most situations (e.g., Theeuwes, 1992), it was expected that RTs would be faster when the target appeared in a singleton rather than in a neutral object. The main interest was whether participants would also show differential RTs when a target appeared in an object consistent rather than inconsistent with the task cue. Such a result would suggest task-based WM guidance of attention.

Method

Participants Thirty-seven University of Canterbury students volunteered for the experiment in exchange for course credit or payment. All of them reported having normal or corrected-to-normal vision.

Apparatus and stimuli Stimuli were presented against a white background on a PC with a 16-in. monitor, and 
E-Prime (Schneider, Eschman, \& Zuccolotto, 2002) was used to generate the stimuli and collect responses. Participants were tested individually in a dimly lit room. The viewing distance was about $60 \mathrm{~cm}$.

The experiment consisted of two parts. Part I was a short shape/gap practice block of 20 trials. Its function was to familiarize the participants with the stimuli and the response keys associated with the object identification task. Part II was the main experiment, in which participants searched for one of two target letters in addition to performing an object identification task. In the shape/gap practice block, each trial consisted of three displays: a task cue, a fixation, and an object display with two geometric shapes (see Fig. 1a). The task cue, which was presented at the center of the screen, was a black word "Shape" or "Gap" written in a bold style in 18-point Courier New font. If the word was "Shape," the task was to report the identity of the red object: whether it was a hexagon or an octagon. If the word was "Gap," the task was to report the location of a gap on the green object: whether the gap was at the top (gap up) or at the bottom (gap down). The fixation was a small black cross of $0.38^{\circ}$ of visual angle at the center of the screen. The object display consisted of two vertically aligned outlined objects with a spatial separation of $1.15^{\circ}$ between their nearest boundaries. One of the objects was red. It was equally likely to be a hexagon or an octagon. The other object was green. It was equally likely to be a rectangle with a gap at the top or at the bottom. On half of the trials, the red object was on top of the green one. On the rest of the trials, their locations were reversed. Each object subtended $2.48^{\circ}$ in length and in width, and the gap of the square was $1.24^{\circ}$.

The trials in the experiment were similar to those in the shape/gap practice block, except that two additional displays were inserted between the fixation and the onset of the object identification display (see Fig. 1b). One of these displays was a fixation cross identical to the one described in the previous paragraph. The other display consisted of four black capital letters, each at the center of an outlined geometric shape. Three of the letters were distractor Xs, and the fourth one was a target letter $\mathrm{M}$ or $\mathrm{W}$. The letters were written in a bold style in 18-point Courier New font. Of the four shapes, three were black circles with a radius of $1.24^{\circ}$. The fourth one was a singleton. It was one of the stimuli associated with the object identification task described above (i.e., a red hexagon or octagon, or a green rectangle with a gap at the top or at the bottom). The four objects were located $3.73^{\circ}$ above, below, to the left of, and to the right of fixation. Participants searched for a target letter $\mathrm{M}$ or $\mathrm{W}$ in the search task.

Design and procedure The experiment had a $2 \times 2$ withinsubjects design, with the principal manipulations being the type of object the letter target was in (singleton vs. neutral) and the relationship of the singleton to the task cue (consistent vs. inconsistent). The letter target was equally likely to appear in any of the four shapes in the search display, and the singleton was consistent or inconsistent with the task cue with equal probability. Thus, the target was in a singleton on one-fourth of the trials and in a singleton consistent with the task cue on one-eighth of the total trials. The four experimental conditions were singleton consistent (SC), where the target was in a singleton consistent with the task cue; singleton inconsistent (SI), where the target was in a singleton inconsistent with the task cue; neutral consistent (NC), where the target was in a neutral object and the singleton in the display was consistent with the task cue; and neutral inconsistent (NI), where the target was in a neutral object and the singleton in the display was inconsistent with the task cue.

In the main experiment, each trial started with a 1,000-ms display of the task cue at the center of the screen, followed by a fixation cross for $1,000 \mathrm{~ms}$. The letter display then appeared for $180 \mathrm{~ms}$. The participants used their right hand to press the "." key, if the target was an M, and the "/" key, if it was a W. Both speed and accuracy were emphasized for the letter task. The response to the target triggered a second fixation cross. After $1,000 \mathrm{~ms}$, the object identification display was shown, and it remained on the screen until a response was made. The participants used their left hand to press one of four labelled keys in accordance with the task cue, pressing " $z$ " for hexagon, " $x$ " for octagon, "c" for gap up, and " $v$ " for gap down. Accuracy, but not speed, was emphasized for the object identification task.

The experiment started with 20 shape/gap practice trials, followed by 20 dual-task practice ones, and then 4 blocks of 80 experimental trials. It took approximately $45 \mathrm{~min}$ to complete.

\section{Results and discussion}

One participant's data were not included in the analyses due to high error rates in the letter task (over $40 \%$ errors in three of the four experimental conditions). Accuracy for the object identification task was high, with an average of $91.7 \%$ correct responses. Performance was slightly better for the gap task (6.7\% error rate) than for the shape task (10\% error rate), although this difference was not statistically significant, $t(35)=1.45, p=.155$.

The main focus of the study was on the letter task. Only those trials where the participants' responses were correct in both the letter discrimination and object identification tasks were analyzed. Table 1 shows the mean RTs. Consistent with prior research (e.g., Theeuwes, 1992), a 
Table 1 Mean reaction times and error rates (percent incorrect), with standard errors, for the letter search task in Experiment 1

\begin{tabular}{|c|c|c|c|c|c|c|c|c|}
\hline & \multicolumn{4}{|c|}{ Singleton } & \multicolumn{4}{|c|}{ Neutral } \\
\hline & \multicolumn{2}{|c|}{ Consistent } & \multicolumn{2}{|c|}{ Inconsistent } & \multicolumn{2}{|c|}{ Consistent } & \multicolumn{2}{|c|}{ Inconsistent } \\
\hline & M & $\mathrm{SE}$ & M & $\mathrm{SE}$ & M & SE & M & $\mathrm{SE}$ \\
\hline RT & 852 & 29.4 & 851 & 31.2 & 900 & 29.1 & 878 & 30.1 \\
\hline \% Error & 7.8 & 0.9 & 9.2 & 1.3 & 11.4 & 1.3 & 11.7 & 1.5 \\
\hline
\end{tabular}

$2 \times 2$ repeated measures ANOVA indicated a main effect of singleton, with faster RTs when the target appeared in a singleton $(852 \mathrm{~ms})$ rather than in a neutral object $(889 \mathrm{~ms})$, $F(1,35)=19.26, M S E=2,639, p<.001$. Moreover, there was a main effect of consistency, with faster RTs when the singleton was inconsistent $(865 \mathrm{~ms})$ rather than consistent (876 ms) with the task cue, $F(1,35)=4.09, M S E=1,129$, $p=.05$. The singleton $\times$ consistency interaction was also significant, $F(1,35)=4.24, M S E=992, p<.05$. Further $t$ tests showed that when the target appeared in the singleton, RTs were comparable regardless of whether the singleton was consistent $(852 \mathrm{~ms})$ or inconsistent $(851 \mathrm{~ms})$ with the task cue, $t(35)=0.05$, n.s. In contrast, when the target appeared in a neutral object, RTs were slower when the singleton in the letter display was consistent $(900 \mathrm{~ms})$ rather than inconsistent $(878 \mathrm{~ms}$ ) with the task cue, $t(35)=3.85$, $p<.001$.

Consistent with the RT results, an ANOVA on the accuracy data showed fewer errors when the target was in a singleton $(8.5 \%$ error rate) rather than in a neutral object (11.6\% error rate), $F(1,35)=6.27, M S E=54, p<.05$. Neither the effect of consistency nor the interaction between consistency and singleton was significant, $F(1,35)=1.69$, $M S E=16, p=.20$, and $F(1,35)<1$, n.s., for the main effect of consistency and the singleton $\times$ consistency interaction, respectively. There was no indication of a speed-accuracy trade-off.

The most important finding in Experiment 1 was that RTs were slower on the consistent than on the inconsistent trials when the target was in a neutral object. This result can be explained in two ways. One is delayed disengagement of attention on the consistent relative to the inconsistent trials. This interpretation assumes that attention is a process that consists of multiple components such as disengagement, movement, and engagement (Posner \& Peterson, 1990). When attention was attracted to a singleton that contained a distractor, it took longer for the participants to disengage attention when the singleton was consistent rather than inconsistent with the task cue. In light of the finding that maintaining an object in WM can increase the activation of the neurons that process the object in many visual areas (e.g., Chelazzi et al., 1993; Gnadt \& Andersen, 1988;
Miller et al., 1996; Rainer et al., 1998; Supèr et al., 2001), it seems reasonable that maintaining a task cue could also enhance the activation level of the stimuli associated with the task, resulting in longer attentional dwell time when disengagement of attention was required.

An alternative interpretation for the differential RTs between the consistent and inconsistent conditions when the target was in a neutral object is stronger attentional capture in the former than the latter condition. If the frequency of the singleton capturing attention was modulated by the contents of WM, or if inhibiting a taskirrelevant singleton required more attentional resources when that singleton was consistent rather than inconsistent with the task cue, RTs would be longer in the NC than in the NI condition. This assumption is consistent with several previous studies. Olivers et al. (2006) reported that their participants made more saccades during a visual search task to a singleton distractor that matched rather than mismatched the memory sample. Soto et al. (2005) also found that their participants' first saccades were more frequently directed to and landed at the location occupied by the item that matched the memory sample. Moreover, Hollingworth and Luck (2009) showed that when their participants had to make a corrective saccade to a target when the first saccade landed between the target and a distractor, accuracy to the target was impaired when the distractor was the memory sample versus when it was not. These results suggest that the contents of WM modulate the frequency, strength, and/ or the direction of saccades. With respect to the present experiment, although our letter display was too brief for participants to make overt eye movements while the target was on (e.g., Alpern, 1972; Mayfrank, Kimmig, \& Fischer, 1987), given the close coupling between saccades and spatial attention (e.g., Deubel \& Schneider, 1996; Hoffman \& Subramaniam, 1995), it is plausible that a singleton that matches the contents of WM can also be more likely to capture spatial attention, regardless of whether overt eye movements are involved. If a cue-consistent singleton attracted covert spatial attention more strongly or more frequently, this would lead to attention being directed to a wrong stimulus more frequently on those trials when the target appeared in a neutral object, resulting in faster RTs in the NI than the NC condition.

In the present experiment, both interpretations are equally likely. However, regardless of the interpretation, we need to answer one question: Why was there no difference in RTs between the SC and SI conditions? Inspection of the letter search display of Experiment 1 suggests that the lack of a task consistency effect on the singleton trials might have been caused by the specific design of the experiment. Recall that a singleton was displayed concurrently with three neutral objects on every trial. When the singleton contained the target, regardless of 
whether it was consistent or inconsistent with the task cue, the singleton was the most salient object in the display and was therefore most likely to attract attention. In other words, because the competition for attention on any given trial was between a singleton and nonsingleton objects instead of between a singleton that was consistent or inconsistent with the task cue, the present design might not have been sensitive enough to detect differences in RTs between the SC and SI conditions.

Experiment 2 was designed to address this problem while holding constant the color of the stimuli between the gap and shape tasks. So far, the shape task had always been associated with red objects, and the gap task with green objects. Although color was irrelevant to the letter discrimination task, the fact that there was a systematic mapping between color and the set of stimuli associated with the shape and gap tasks could induce participants to use color as a mnemonic cue, and in doing so, use it to guide attention during visual search. Given that previous research has shown that color can guide attention in visual search (e.g., Bacon \& Egeth, 1997; Carter, 1982) and that holding the verbal description of a colored geometric shape (e.g., "Red Square") in mind can also influence the distribution of attention (e.g., Soto \& Humphreys, 2007), it is possible that the systematic relationship between color and the stimulus set in the shape and gap tasks contributed to the WM effect found in Experiment $1^{1}$.

\section{Experiment 2}

Experiment 2 tested the effect of task-based WM on attention while holding constant the color of the stimuli in the gap and shape tasks. Unlike in Experiment 1, the letter display consisted of two geometric shapes: one consistent and the other inconsistent with the task cue. Since there was no singleton in the search display, the competition for attention was between two items of equal physical salience. To render color unhelpful, we changed it so that all the geometric shapes were shown in red ink in the letter discrimination display, but in black ink in the object identification display. We also reduced the size of the shapes in the object identification display. By making the stimuli differ in color and size between the two displays, we hoped to discourage participants from conjuring up cueconsistent images and using them to guide attention in the letter task. From a participant's perspective, the behavioral significance of the task cue was its relevance to the object identification task. When the stimuli in the object identification display differed from those in the letter display, even

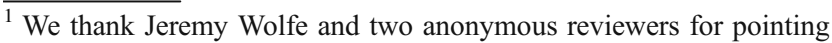
this out.
}

if cue-consistent images were evoked on some of the trials, the stimuli evoked would be those associated with the object task instead of with the letter task. Given the new design in Experiment 2, a cue consistency effect in the letter discrimination task would support the notion of a tight link between task-based WM and the distribution of visual attention.

\section{Method}

The method of Experiment 2 was the same as that of Experiment 1, except for the following differences (see Fig. 2). First, the letter display consisted of two horizontally aligned red objects, each containing a black letter inside. The objects, one consistent and the other inconsistent with the task cue, were located $6.02^{\circ}$ to the left and to the right of fixation, and their locations were counterbalanced across the trials. The letter display was shown for $160 \mathrm{~ms}$. Second, whereas one of the letters was equally likely to be a target letter $\mathrm{M}$ or $\mathrm{W}$, the other was always a distractor X. On half of the trials, the target letter was in the object consistent with the task cue (the consistent condition). On the rest of the trials, it was in the object inconsistent with the task cue (the inconsistent condition). Finally, the two geometric shapes in the object identification display were black, and each of them subtended $1.72^{\circ}$ in length and in width. Twenty-two new participants took part in the experiment.

\section{Results and discussion}

Accuracy for the object identification task was again very high, with an average of $96.1 \%$ correct. As in Experiment 1 , there was no significant difference between the gap and shape tasks (4.9\% vs. $5.8 \%$ error rates for the gap and the shape tasks, respectively), $t(21)=1.11$, n.s.

For the letter search task, 2 participants' data were excluded from analyses due to their long RTs (one more than 3 and the other 2.5 standard deviations from the mean of the rest of the participants). As in Experiment 1, only those trials where the participants' responses were correct in both the letter discrimination and object identification tasks were analyzed. There was no significant difference between the consistent ( $748 \mathrm{~ms}$ with $6.8 \%$ error rate) and inconsistent (739 $\mathrm{ms}$ with $7.1 \%$ error rates) conditions in either RTs or accuracy, $t(19)<1$ in both cases. However, visual inspection of the data revealed that the participants whose RTs were fast showed a different pattern of data from those whose RTs were slow. To explore this further, we divided the participants into two groups based on their RTs. The fastest-responding half of the participants across the consistent and inconsistent conditions were assigned to the fast group, and the rest of them to the slow group. The results are shown in Table 2. A repeated measures ANOVA 


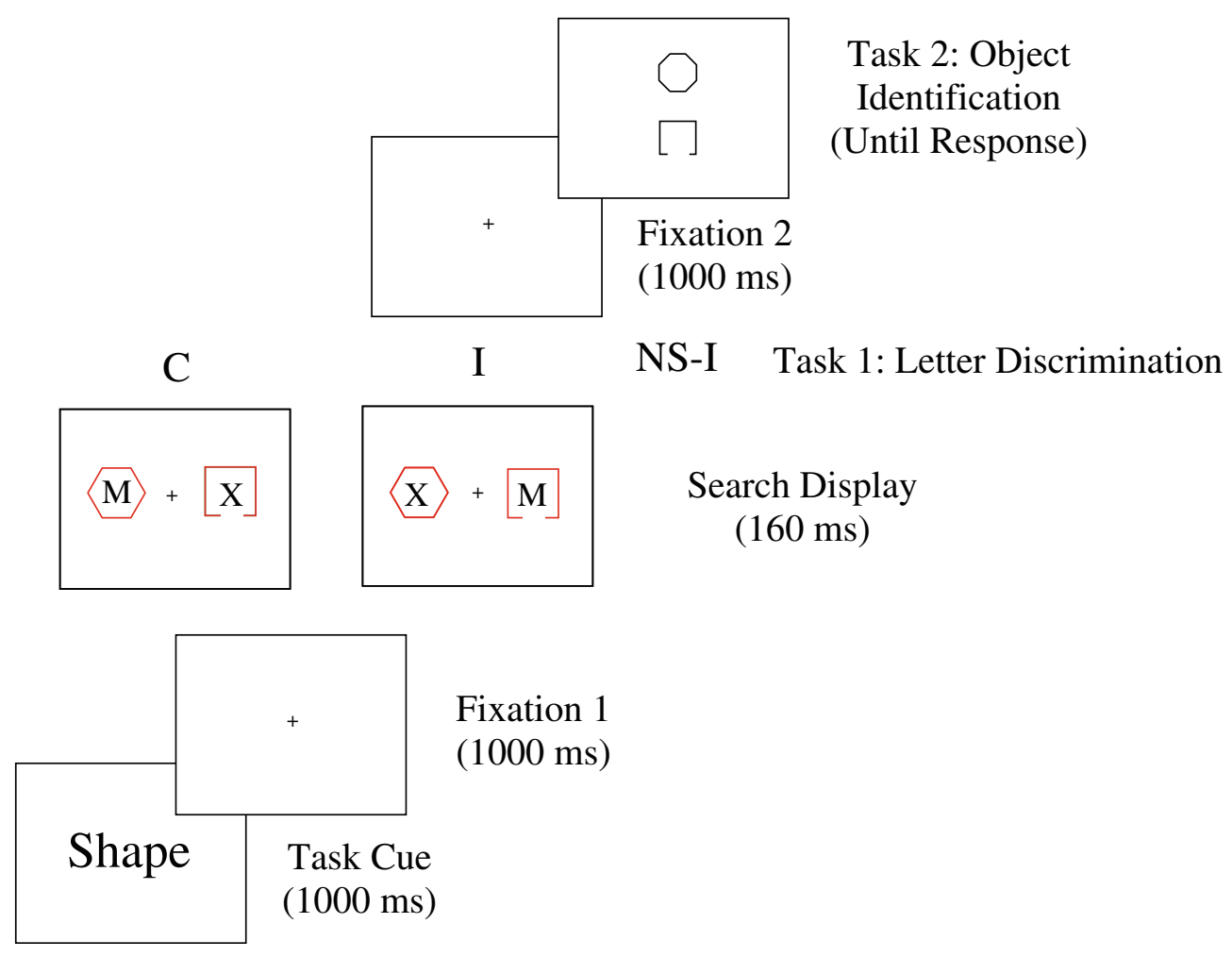

Fig. 2 Examples of stimulus displays from Experiment 2. Whereas both the geometric shapes were red in the search display, they were black and smaller in the object identification display. $\mathrm{C}$ refers to the consistent condition, and I refers to the inconsistent condition

on RTs with group as a between-subjects factor and consistency as a within-subjects factor indicated a significant main effect of group, $F(1,18)=38.16, M S E=9,555$, $p<.001$. As expected, RTs were faster in the fast group (648 $\mathrm{ms}$ ) than in the slow group (839 ms). Interestingly, although there was no main effect of consistency, $F(1,18)=$ 1.0, n.s., there was a significant group $\times$ consistency interaction, $F(1,18)=5.07, M S E=696, p<.05$. Subsequent $t$ tests indicated a task consistency effect for the slow group, $t(9)=2.07, p<.05$, but not for the fast group, $t(9)=-1.01$, n.s. These results suggest that whereas the participants in the slow group took longer to perform the letter discrimination task when the target occurred in a cue-consistent object (852 ms) compared with a cue-inconsistent object (825 ms), a similar effect was not found for the participants in the fast group (643 ms and $653 \mathrm{~ms}$ for the consistent and

Table 2 Mean reaction times and error rates (percent incorrect), with standard errors, for the letter search task in Experiment 2

\begin{tabular}{|c|c|c|c|c|c|c|c|c|}
\hline & \multicolumn{4}{|c|}{ Fast Group } & \multicolumn{4}{|c|}{ Slow Group } \\
\hline & \multicolumn{2}{|c|}{ Consistent } & \multicolumn{2}{|c|}{ Inconsistent } & \multicolumn{2}{|c|}{ Consistent } & \multicolumn{2}{|c|}{ Inconsisten } \\
\hline & M & SE & M & SE & M & $\mathrm{SE}$ & M & SE \\
\hline RT & 643 & 22.3 & 653 & 20.3 & 852 & 21.5 & 825 & 26.3 \\
\hline$\%$ Error & 7.8 & 2.3 & 7.0 & 2.0 & 5.8 & 1.2 & 7.2 & 1.9 \\
\hline
\end{tabular}

inconsistent conditions, respectively). There were no significant effects on the accuracy data.

The results of Experiment 2 suggest two tentative conclusions. First, color indeed contributed to the results of Experiment 1. This was evidenced by the finding that the effect of WM was eliminated for the participants in the fast group in Experiment 2 when color was held constant across the stimuli in the shape and gap tasks. In Experiment 1, although the specific task cue held in memory was irrelevant to the letter discrimination task, the fact that there was a systematic mapping between color and the target stimulus in the object identification task may have induced the participants, at least on some of the trials, to hold color instead of the task cue in WM, resulting in the cue consistency effect found in the letter discrimination task. Second, when color was held constant, the contents of WM still influenced the distribution of attention for those participants whose response latencies were relatively long. Since neither color nor cue-consistent images could contribute to the guidance of attention in Experiment 2, the finding of the cue consistency effect in the slow group is consistent with the notion of task-based WM guidance of attention, suggesting that the effect of WM on attention could be generalized from a specific stimulus to a task, at least under some circumstances.

One may wonder whether the direction of the cueconsistent effect in the slow group (i.e., slower RTs in the consistent than in the inconsistent condition) is consistent 
with object-based deployment of attention. Research on object-based attention has shown that features that belong to the same object or perceptual group are processed more efficiently than features that belong to different objects or perceptual groups (e.g., Chen, 1998; Duncan, 1984; Egly, Driver, \& Rafal, 1994; Kramer \& Jacobson, 1991). Note, however, this same-object advantage appears typically in situations where the features are perceived as part of the same object. In other words, we would expect the participants in the present experiment to have faster RTs in the consistent than in the inconsistent condition if the letter and its surrounding shape in the letter display were perceived as part of the same object or perceptual group, and more importantly, if the presence of the shapes did not interfere with the processing of the letters.

The fact that RTs were slower in the consistent than in the inconsistent condition suggests that the presence of the geometric shapes in the letter display interfered with the letter discrimination task. Previous research has shown that participants take longer to respond to a target flanked by distractors when the target and distractors appear on the same object rather than when they appear on different objects, and this phenomenon has been attributed to a segmentation process that participants evoke to separate the target from the distractors when they occur on the same object (Chen \& Cave, 2006; Shomstein \& Yantis, 2002).

To empirically verify the effect of geometric shape on letter discrimination, we conducted a simple experiment with 4 participants (Z.C. and three naïve students). The main manipulation was the presence or absence of geometric shapes in a letter display. Each trial started with a 1,000-ms fixation cross followed by a pair of letters (an X with either an $\mathrm{M}$ or a W) for $160 \mathrm{~ms}$. The task was to determine, as quickly and as accurately as possible, whether an $\mathrm{M}$ or a $\mathrm{W}$ was present. Each participant completed two blocks of 128 trials. In one block, the letters were placed inside geometric shapes (identical to the letter discrimination display in Experiment 2). In the other block, no shapes were present. The order of the blocks was counterbalanced across participants. The results showed that all 4 of the participants had longer RTs when the shapes were present than when they were absent, with their mean RTs being $495 \mathrm{~ms}$ on the shape-present trials and $430 \mathrm{~ms}$ on the shape-absent trials, $t(3)=6.37, p<.01$. Furthermore, 3 of the 4 participants also made more errors on the shapepresent trials than on the shape-absent trials, and the mean error rates for the 4 participants were $9.4 \%$ and $2.5 \%$ for the shape-present and shape-absent trials, respectively. These results confirmed that the presence of the shapes interfered with the processing of the letters and delayed the participants' responses in the letter discrimination task.

In light of the above results, one interpretation for the differential WM effects between the fast and slow groups was that the two groups differed in their ability to inhibit irrelevant information. If we consider RTs as a valid measure of processing efficiency, it seems reasonable to assume that, on average, the participants with fast RTs are efficient information processors who can inhibit irrelevant distractors more effectively than those with slower RTs. Since the geometric shapes in the letter display were irrelevant distractors that interfered with the processing of the target, their effect should be less on those with high inhibitory ability than on those with low inhibitory ability. Consequently, whereas the fast group did not show the cue consistency effect, the slow group took longer to perform the letter discrimination task in the consistent than in the inconsistent condition.

It should be noted that our results differed from those of Han and Kim (2009, Exp. 1). In their experiment, the participants with fast RTs showed an enhanced WM effect while the participants with slow RTs showed a reduced WM effect. There are many methodological differences between the Han and Kim study and our Experiment 2. These differences, which include the relationship between the content of WM and the target-defining feature in visual search (part of the same object vs. different objects), the nature of the attention task and task demand (difficult location discrimination vs. easy letter discrimination), memory requirement (object feature vs. task cue), and the method of memory assessment (recognition vs. object identification), make it unclear what caused the different results between the two studies. Regardless of the nature of the differences, one thing is clear: the effect of WM guidance on attention is more complex than was previously understood. It can be influenced by participants' response latencies, which in turn may be associated with differences in cognitive control abilities, cortical efficiencies, and perhaps response strategies between the fast and slow participants (see also Kosslyn, Thompson, Kim, Rauch, \& Alpert, 1996, and Rypma \& D’Esposito, 1999, for evidence of correlations between changes in cerebral blood flow and participants' RTs in tasks that required WM maintenance, attention, and mental image generation).

Admittedly, our interpretation of the group differences in Experiment 2 was post hoc. To test our hypothesis, we need to have an independent measure to examine whether the participants who had fast RTs were indeed better at inhibiting irrelevant information than those who had slow RTs. We addressed this issue in Experiment 3B, after we replicated the results of Experiment 2 in Experiment $3 \mathrm{~A}$.

\section{Experiments $3 \mathrm{~A}$ and $3 \mathrm{~B}$}

Experiments $3 \mathrm{~A}$ and $3 \mathrm{~B}$ had two goals: to replicate the results of Experiment 2 in Experiment 3A, and to use the 
magnitude of Stroop interference in Experiment 3B as an independent measure of distractor inhibition to determine whether the participants with fast RTs in Experiment 3A would inhibit irrelevant information more effectively than their counterparts with slow RTs.

\section{Experiment 3A}

In Experiment 3A, we sought to replicate the results of Experiment 2 with a slightly different design. Instead of presenting all the stimuli in the visual search display simultaneously, we delayed the onset of the letters for $120 \mathrm{~ms}$ after the appearance of the shapes, so that the contents of WM would not have to compete with the letters immediately. Without the competition from the letters, a cue-consistent shape would only need to compete with a cue-inconsistent shape, and this might increase the sensitivity of the experiment if the cue-consistent shape was more likely to attract attention, and therefore required more attentional resources to inhibit. We also decreased the size of the letters, making them more difficult to identify without focal attention. The reduction in size was to induce participants to make overt eye movements. If the effect of WM was again found with overt eye movements, this would provide converging evidence for the results of Experiment 2.

\section{Method}

The method of Experiment 3A was the same as that of Experiment 2, except that the letters in the search display appeared $120 \mathrm{~ms}$ after the onset of the geometric shapes, and their size and font style changed from 18-point bold to 8-point regular. The letter search display stayed on the screen until response. Twenty new undergraduates from the same participant pool volunteered for the experiment.

\section{Results and discussion}

Once again, the accuracy of the object identification task was very high, and there was no significant difference between the gap and shape tasks $(5.6 \%$ vs. $5.0 \%$ error rates for the gap and the shape task, respectively), $t(19)=$ 0.57 , n.s.

For the letter search task, as in Experiment 2, we first analyzed the participants' RT and accuracy data as a whole group. Again, only the correct trials were included in the RT analyses. There was no significant difference between the consistent condition ( $828 \mathrm{~ms}$ with $3.2 \%$ error rate) and the inconsistent condition ( $821 \mathrm{~ms}$ with $4.0 \%$ error rates) in either RTs, $t(19)=1.33$, n.s., or accuracy, $t(19)=-1.52$, n.s. However, when we divided the participants into a fast and a slow group based on their mean response latencies in the search task, the two groups again showed different patterns of data (see Table 3). Not surprisingly, responses were faster in the fast group (756 ms) than in the slow group $(894 \mathrm{~ms}), F(1,18)=24.34, M S E=7,841, p<.001$. Importantly, there was a significant interaction between group and consistency, $F(1,18)=5.96, M S E=228, p<$ .05 . As in Experiment 2, whereas the participants in the slow group had longer RTs in the consistent condition $(903 \mathrm{~ms})$ than in the inconsistent condition $(884 \mathrm{~ms}), t(9)=$ $2.72, p<.05$, the participants in the fast group did not show a cue consistency effect (753 and $758 \mathrm{~ms}$ for the consistent and inconsistent conditions, respectively), $t(9)=-0.68$, n.s. There were no significant effects in the accuracy data.

The results of Experiment 3A were similar to those of Experiment 2. In both experiments, only those participants who were relatively slow in visual search were influenced by the contents of WM, and their response latencies were delayed when the target appeared in the object consistent rather than inconsistent with the task cue $^{2}$. In the next experiment, we used a Stroop paradigm to determine whether the participants who responded fast versus slow in Experiment $3 \mathrm{~A}$ would also differ in the magnitude of Stroop interference.

\section{Experiment 3B}

Experiment 3B used a Stroop interference paradigm (Stroop, 1935) to assess the participants' ability to inhibit irrelevant information (see MacLeod, 1991, for a review). By recruiting the same people who took part in Experiment $3 \mathrm{~A}$ to participate in Experiment 3B, we could assess whether the participants with long RTs in Experiment 3A would show larger Stroop interference than the participants with short RTs. The degree of Stroop interference has often been taken as a valid measure of one's ability to inhibit irrelevant information. It has been shown that Stroop interference is larger in older adults than in younger ones (e.g., Cohn, Dustman, \& Bradford, 1984; Hartley, 1993), and inhibitory function is known to decline with aging

\footnotetext{
${ }^{2}$ Is it possible that the WM effect on attention in Experiments 2 and 3 shown by the slow participants was caused by the word "gap" acting as a cue for "square"? Although it is difficult to rule that possibility out entirely without further experiments, its likelihood seems quite small. If "gap" had acted as a cue for "square" (and "shape" as a cue for "nonsquare"), we would expect "gap" to be a more powerful cue than "shape," and this in turn should have led to a greater WM effect on attention in the gap task than in the shape task. However, the data from Experiments 2 and $3 \mathrm{~A}$ do not appear to reflect that. In Experiment 2, the effect of WM on attention was shown primarily in the shape task (the difference between the consistent and inconsistent conditions was $52 \mathrm{~ms}$ ), but not in the gap task (the difference was $1 \mathrm{~ms}$ ). In Experiment $3 \mathrm{~A}$, the effects of WM were comparable in the two tasks (the difference between the consistent and inconsistent conditions was $18 \mathrm{~ms}$ in the shape task and $21 \mathrm{~ms}$ in the gap task).
} 
Table 3 Mean reaction times and error rates (percent incorrect), with standard errors, for the letter search task in Experiment 3A

\begin{tabular}{|c|c|c|c|c|c|c|c|c|}
\hline & \multicolumn{4}{|c|}{ Fast Group } & \multicolumn{4}{|c|}{ Slow Group } \\
\hline & \multicolumn{2}{|c|}{ Consistent } & \multicolumn{2}{|c|}{ Inconsistent } & \multicolumn{2}{|c|}{ Consistent } & \multicolumn{2}{|c|}{ Inconsisten } \\
\hline & M & SE & M & $\mathrm{SE}$ & M & $\mathrm{SE}$ & M & SE \\
\hline RT & 753 & 16.3 & 758 & 15.3 & 903 & 24.6 & 884 & 22.7 \\
\hline$\%$ Error & 3.4 & 0.7 & 4.5 & 1.0 & 3.0 & 0.6 & 3.5 & 0.7 \\
\hline
\end{tabular}

(e.g., Dempster, 1992; McDowd \& Oseas-Kreger, 1991). In terms of the present study, if the participants in the slow group of Experiment 3A showed a larger Stroop interference effect than those in the fast group, this would support the notion that the two groups were indeed different in their ability to inhibit irrelevant information, and that this difference gave rise to the differential effects of WM found in the present study.

\section{Method}

Participants Fifteen out of the original 20 participants who took part in Experiment 3A participated in the present experiment. Each was paid NZ\$10 for their participation. The other 5 students (4 from the fast group, and 1 from the slow group) did not show up for the experiment, despite our repeated attempts to invite them back.

Apparatus and stimuli The apparatus was the same as that used in the previous experiments. All stimuli were presented against a gray background. Each trial consisted of a fixation cross followed by a target. The fixation, which was shown at the center of screen, was a black cross of $0.48^{\circ}$ visual angle. The target was a colored stimulus. The four colors used in the experiment were red, green, yellow, and blue. On incongruent trials, the stimulus was a word (i.e., red, green, yellow, or blue) in one of three colors other than the color that matched its meaning. On neutral trials, it was a string of letters corresponding in length (i.e., $v v v$, sssss, nnnnnn, or oooo) written in colored ink. For example, for the stimulus red or its corresponding letter string $v v v$, the color was equally likely to be green, yellow, or blue, but not red. On half of the trials, the target stimulus appeared $0.86^{\circ}$ above the fixation cross. On the rest of the trials, it appeared at a corresponding location below fixation.

Design and procedure The experiment had a withinsubjects design. The principal manipulation was the relationship between the meaning of the stimulus and the ink color (incongruent vs. neutral). There were as many incongruent trials as neutral ones.
Each trial started with the display of the fixation for $1,000 \mathrm{~ms}$. Upon its offset, the target appeared either above or below the center of the screen with equal probability and remained there until a response was made. The task was to identify the color of the target by pressing one of the four labelled keys on the keyboard. The participants used the middle and forefingers of both hands to press one of four labelled keys on the keyboard (" $\mathrm{z}$ " for red, " $\mathrm{x}$ " for green, "." for yellow, and " " for blue). Both speed and accuracy were emphasized. The experiment consisted of 30 practice trials, followed by three blocks of 96 trials. The entire experiment took approximately $15 \mathrm{~min}$ to complete.

\section{Results and discussion}

Since the main interest of Experiment 3B was to determine whether the participants with slow RTs in Experiment 3A would show greater Stroop interference than those with fast RTs, we divided the participants into two groups (fast vs. slow) based on their RTs in Experiment 3A. The results are shown in Table 4. As expected, there were a significant Stroop interference effect and a group effect on RTs. The participants took longer to respond when the color of the ink was incongruent with the meaning of the word $(730 \mathrm{~ms})$ than when the two were unrelated $(664 \mathrm{~ms}), F(1,13)=$ 65.13, MSE $=433, p<.0001$. RTs were also longer for the participants in the slow group (743 ms) than for those in the fast group (630 ms), $F(1,13)=7.33, M S E=12,333, p<$ .05. Importantly, the magnitude of the Stroop interference effect was larger in the slow group $(79 \mathrm{~ms})$ than in the fast group (46 ms), $F(1,13)=4.60, M S E=433, p=.05$. No significant effects were found on the accuracy data ${ }^{3}$.

The finding of the group difference in the magnitude of Stroop interference in Experiment 3B is consistent with the notion that participants who differed in response latencies also differed in their ability to inhibit task-irrelevant information, and the latter difference was likely to have led to the differential WM effects on attention that we observed between the fast and slow groups of participants in Experiments 2 and 3A.

\section{General discussion}

Previous research established that keeping a stimulus in WM can influence the deployment of visual attention (e.g.,

\footnotetext{
${ }^{3}$ The correlation between the participants' RTs in the letter discrimination task in Experiment $3 \mathrm{~A}$ and the amount of Stroop interference they showed in Experiment $3 \mathrm{~B}$ was $.43, p=.108$. Although the correlation was not significant, given the small number of participants who took part in both experiments $(N=15)$, this result was not surprising.
} 
Table 4 Mean reaction times and error rates (percent incorrect), with standard errors, for the Stroop task in Experiment 3B

\begin{tabular}{|c|c|c|c|c|c|c|c|c|}
\hline & \multicolumn{4}{|c|}{ Fast Group } & \multicolumn{4}{|c|}{ Slow Group } \\
\hline & \multicolumn{2}{|c|}{ Incongruent } & \multicolumn{2}{|c|}{ Neutral } & \multicolumn{2}{|c|}{ Incongruent } & \multicolumn{2}{|c|}{ Neutral } \\
\hline & M & SE & M & SE & M & $\mathrm{SE}$ & M & SE \\
\hline RT & 653 & 29.6 & 607 & 24.3 & 782 & 30.4 & 703 & 27.8 \\
\hline$\%$ Error & 4.9 & 0.8 & 5.3 & 1.0 & 3.9 & 1.2 & 3.3 & 0.7 \\
\hline
\end{tabular}

The participants were assigned to one of the two groups based on their RTs in Experiment 3A.

Downing, 2000; Huang \& Pashler, 2007; Moores et al., 2003; Olivers et al., 2006; Pashler \& Shui, 1999; Soto et al., 2005). The present experiments show that maintaining a task in WM can also influence the strength and/or the frequency of attentional capture of the stimuli associated with the task. Moreover, this task-based WM guidance on attention was modulated by participants' response latencies. Whereas the participants with fast RTs showed little effect of WM contents, those with slow RTs took longer to respond when the target appeared in an object consistent rather than inconsistent with the task held in mind. Using the magnitude of Stroop interference as an index of distractor interference, we found a larger Stroop effect for those with slow RTs than for those with fast RTs. These results suggest that the two groups differed in their ability to inhibit task-irrelevant information, and that this difference gave rise to the differential WM effects between the fast and slow participants in our experiments.

Our results add to a growing number of studies that have shown the complexity of the WM effect on attention. WM guidance of attention appears to be more likely to occur when only one or two objects are to be held in mind (Soto \& Humphreys, 2008; Woodman \& Luck, 2007); when the salience of the memory sample is high (Olivers, 2009); when the variability of the target across trials is low (Downing \& Dodds, 2004; Olivers, 2009); when the level of cognitive control that can be exerted over the contents of WM is low (Han \& Kim, 2009); and when the coding of the memory sample is visual rather than verbal (e.g., Olivers et al., 2006; but see Olivers, 2009; Soto et al., 2005; Soto \& Humphreys, 2007; and the present experiments for evidence of verbal coding). Our finding in the present study that the effect of task-based WM on attention disappeared for a substantial number of participants when the covariation of color with the task cue was eliminated in Experiments 2 and $3 \mathrm{~A}$ also demonstrated the relative fragility of task-based WM guidance of attention compared with feature-based WM guidance of attention. Taken together, the available evidence shows a close link between attention and WM, although the manifestation of the effect of WM guidance on attention depends on many factors.

\section{Mechanisms of task-based WM guidance of attention}

We interpret the task-based WM guidance of attention in the framework of the biased competition model of attention (Desimone, 1998; Desimone \& Duncan, 1995). According to the model, attention is best understood "in the context of competition among all of the stimuli in the visual field for control of behaviour" (Desimone, 1998, p. 1245). When a display contains multiple objects, their neural representations compete for access to higher-level processing and the control of behavior. This competition can be biased by many factors from both bottom-up and top-down processes. In terms of the present experiments, when a task was maintained in WM, stimuli associated with the task were activated, and this in turn gave them an advantage relative to other stimuli in the competition for attention. However, because these stimuli were distractors that interfered with the processing of the target in visual search, the increased activity made them harder to ignore when they were in the same object as the target relative to when the two sets of stimuli were in different objects. This was especially the case for those participants who were slow in evoking inhibition and/or were relatively weak in inhibitory mechanisms. The difference in inhibitory abilities between the fast and slow participants may have been related to differences in the function of the frontal lobes, which are known to be involved in the inhibition of irrelevant stimuli and in WM maintenance (e.g., Awh \& Jonides, 2001; Konishi et al., 1999; Milner, 1964). There is evidence that participants with fast and slow RTs differ in the activation of the dorsal prefrontal cortex (Rypma \& D'Esposito, 1999) and that, relative to normal controls, patients with frontallobe damage show a larger WM effect on the distribution of attention (Soto, Humphreys, \& Heinke, 2006).

The neural circuits involved in the task-based WM guidance of attention are likely to include many of the same cortical areas that are involved in attention and WM maintenance (for reviews, see Awh \& Jonides, 2001; Postle, 2006). Since the task in our experiments emphasized the maintenance of object information, these areas were likely to include, among other brain regions, the inferior temporal cortex and the prefrontal cortex. The inferior temporal cortex is known to be involved in the processing of objects and object features (e.g., Farah, 1991; Grady et al., 1991; Smith et al., 1995), and there is evidence that the prefrontal cortex participates in the maintenance of object information in WM (e.g., Courtney, Ungerleider, Keil, \& Haxby, 1997; Wilson, Scalaidhe, \& Goldman-Rakic, 1993). In addition to these brain areas, other high-level regions that were likely to be involved in the present experiments 
include the left frontal speech regions (i.e., Broca's area, the premotor area, and the supplementary motor area) and the left posterior parietal cortex. It has been shown that these brain areas are actively involved in the performance of verbal WM tasks (e.g., Awh, Smith, \& Jonides, 1995; Paulesu, Frith, \& Frackowiak, 1993; Schumacher et al., 1996). In the present study, the participants were required to memorize the task cue, and postexperiment interviews with them indicated that they used verbal rehearsal to hold the task cue in mind.

Stimulus specificity and WM guidance of attention

Our results suggest that WM guidance of attention does not have to be stimulus-specific. Although we used only two stimuli in each task, and therefore cannot on their own generalize these results to tasks associated with a large stimulus set, the idea of WM guidance of attention by a concept rather than a specific stimulus is not new. Several researchers (e.g., Dark, Vochatzer, \& Van Voorhis, 1996; Moores et al., 2003; Stolz, 1996; Vogt, De Houwer, Moors, Van Damme, \& Crombez, 2010) have shown that semantic relatedness can influence the distribution of attention. Dark et al. found that when participants were only able to report one of two words, they were more likely to report the word that was semantically related rather than unrelated to a previously presented word. Stolz showed that the cost of an invalid spatial cue was larger when the cue consisted of a pair of semantically related rather than unrelated words. Moores et al. further demonstrated the guidance of attention by associative knowledge in long-term memory. Their participants searched for a pictorial target indicated by a verbal precue and then recalled as many objects from the search display as possible. The results indicated that the participants were more likely to recall an object associated with the verbal cue (e.g., a picture of a helmet when the cue was "Motorbike") than to recall a neutral object. More recently, Vogt et al. (2010) reported that the guidance of attention by associative knowledge occurred only when the stimuli held in WM were linked directly to participants' behavioral goals. Taken together, these results indicate that for WM contents to influence attention, they do not have to be stimulus-specific, although they do need to be linked to the participants' behavioral goals.

To conclude, the present experiments provide further evidence for a close link between attention and WM. They suggest that WM's guidance of attention does not have to be stimulus-specific. Stimuli that are associated with a task can also influence the deployment of visual attention. However, whether the increased activation of these stimuli impairs performance when they are task irrelevant may depend on the participants' ability to inhibit irrelevant information.
Author Note We thank Jeremy Wolfe, Michael Kane, and three anonymous reviewers for their helpful comments.

\section{References}

Alpern, M. (1972). Eye movements. In D. Jameson \& L. M. Hurvich (Eds.), Handbook of sensory physiology (Vol. 7, pp. 303-330). Berlin: Springer

Awh, E., \& Jonides, J. (2001). Overlapping mechanisms of attention and spatial working memory. Trends in Cognitive Science, 5, 119-126. doi:10.1016/S1364-6613(00)01593-X

Awh, E., Smith, E. E., \& Jonides, J. (1995). Human rehearsal processes and the frontal lobes: PET evidence. In J. Grafman, K. Holvoak, \& F. Boller (Eds.), Structure and functions of the human prefrontal cortex (Annals of the New York Academy of Sciences, Vol. 769, pp. 97-119). New York: New York Academy of Sciences. doi:10.1111/j.1749-6632.1995.tb38134.x

Bacon, W. F., \& Egeth, H. E. (1997). Goal-directed guidance of attention: Evidence from conjunctive visual search. Journal of Experimental Psychology: Human Perception and Performance, 23, 948-961. doi:10.1037/0096-1523.23.4.948

Bundesen, C. (1990). A theory of visual attention. Psychological Review, 97, 523-547

Cabeza, R., \& Nyberg, L. (2000). Imaging cognition II: An empirical review of 275 PET and fMRI studies. Journal of Cognitive Neuroscience, 12, 1-47. doi:10.1162/08989290051137585

Carter, R. C. (1982). Visual search with color. Journal of Experimental Psychology: Human Perception and Performance, 8, 127136. doi:10.1037/0096-1523.8.1.127

Chelazzi, L., Miller, E. K., Duncan, J., \& Desimone, R. (1993). A neural basis for visual search in inferior temporal cortex. Nature, 363, 345-347. doi:10.1038/363345a0

Chen, Z. (1998). Switching attention within and between objects: The role of subjective organization. Canadian Journal of Experimental Psychology, 52, 7-16. doi:10.1037/h0087274

Chen, Z., \& Cave, K. R. (2006). Reinstating object-based attention under positional certainty: The importance of subjective parsing. Perception \& Psychophysics, 68, 992-1003

Cohn, J. D., Dustman, R. E., \& Bradford, D. C. (1984). Age-related decrements in Stroop color test performance. Journal of Clinical Psychology, 40, 1244-1250. doi:10.1002/1097-4679(198409

Courtney, S. M., Ungerleider, L. G., Keil, K., \& Haxby, J. V. (1997). Transient and sustained activity in a distributed neural system for human working memory. Nature, 386, 608-611. doi:10.1038/ $386608 \mathrm{a} 0$

Dark, V. J., Vochatzer, K. G., \& Van Voorhis, B. A. (1996). Semantic and spatial components of selective attention. Journal of Experimental Psychology: Human Perception and Performance, 22, 63-81. doi:10.1037/0096-1523.22.1.63

Dempster, F. N. (1992). The rise and fall of the inhibitory mechanism: Toward a unified theory of cognitive development and aging. Developmental Review, 12, 45-75. doi:10.1016/0273-2297(92) 90003-K

Desimone, R. (1998). Visual attention mediated by biased competition in extrastriate visual cortex. Philosophical Transactions of the Royal Society B, 353, 1245-1255. doi:10.1098/rstb.1998.0280

Desimone, R., \& Duncan, J. (1995). Neural mechanisms of selective visual attention. Annual Review of Neuroscience, 18, 193-222. doi:10.1146/annurev.ne.18.030195.001205

Deubel, H., \& Schneider, W. X. (1996). Saccade target selection and object recognition: Evidence for a common attentional mechanism. Vision Research, 36, 1827-1837. doi:10.1016/0042-6989 (95)00294-4 
Downing, P. E. (2000). Interactions between visual working memory and selective attention. Psychological Science, 11, 467-473. doi:10.1111/1467-9280.00290

Downing, P. E., \& Dodds, C. M. (2004). Competition in visual working memory for control of search. Visual Cognition, 11, 689-703. doi:10.1080/13506280344000446

Duncan, J. (1984). Selective attention and the organization of visual information. Journal of Experimental Psychology: General, 113, 501-517. doi:10.1037/0096-3445.113.4.501

Egeth, H. E., \& Yantis, S. (1997). Visual attention: Control, representation and time course. Annual Review of Psychology, 48, 269-297. doi:10.1146/annurev.psych.48.1.269

Egly, R., Driver, J., \& Rafal, R. D. (1994). Shifting visual attention between objects and locations: Evidence from normal and parietal lesion subjects. Journal of Experimental Psychology: General, 123, 161-177. doi:10.1037/0096-3445.123.2.161

Farah, M. J. (1991). Patterns of co-occurrence among the associative agnosias: Implications for visual object recognition. Cognitive Neuropsychology, 8, 1-19

Gnadt, J. W., \& Andersen, R. A. (1988). Memory related motor planning activity in posterior parietal cortex of macaque. Experimental Brain Research, 70, 216-220. doi:10.1007/BF00271862

Grady, C. L., Haxby, J. V., Horwitz, B., Ungerleider, L. G., Shapiro, M. B., Carson, R. E., et al. (1991). Dissociation of object and spatial vision in human extrastriate cortex. Journal of Cognitive Neuroscience, 4, 23-24

Han, S. W., \& Kim, M.-S. (2009). Do the contents of working memory capture attention? Yes, but cognitive control matters. Journal of Experimental Psychology: Human Perception and Performance, 35, 1292-1302. doi:10.1037/a0016452

Hartley, A. A. (1993). Evidence for the selective preservation of spatial selective attention in old age. Psychology and Aging, 8, 371-379. doi:10.1037/0882-7974.8.3.371

Hoffman, J. E., \& Subramaniam, B. (1995). The role of visual attention in saccadic eye movements. Perception \& Psychophysics, 57, 787-795

Hollingworth, A., \& Luck, S. J. (2009). The role of visual working memory (VWM) in the control of gaze during visual search. Attention, Perception, \& Psychophysics, 71, 936-949. doi:10.3758/APP.71.4.936

Huang, L., \& Pashler, H. (2007). Working memory and the guidance of visual attention: Consonance-driven orienting. Psychonomic Bulletin \& Review, 14, 148-153

Kastner, S., De Weerd, P., Desimone, R., \& Ungerleider, L. G. (1998). Mechanisms of directed attention in the human extrastriate cortex as revealed by functional MRI. Science, 282, 108-111. doi:10.1126/science.282.5386.108

Konishi, S., Nakajima, K., Uchida, I., Kikyo, H., Kameyama, M., \& Miyashita, Y. (1999). Common inhibitory mechanism in human inferior prefrontal cortex revealed by event-related functional MRI. Brain, 122, 981-991. doi:10.1093/brain/122.5.981

Kosslyn, S. M., Thompson, W. I., Kim, I. J., Rauch, S. I., \& Alpert, N. M. (1996). Individual differences in Area 17 predict the time to evaluate visualized letters. Journal of Cognitive Neuroscience, 8 , 78-82. doi:10.1162/jocn.1996.8.1.78

Kramer, A. F., \& Jacobson, A. (1991). Perceptual organization and focused attention: The role of objects and proximity in visual processing. Perception \& Psychology, 50, 267-284

MacLeod, C. M. (1991). Half a century of research on the Stroop effect: An integrative review. Psychological Bulletin, 109, 163203. doi:10.1037/0033-2909.109.2.163

Mayfrank, L., Kimmig, H., \& Fischer, B. (1987). The role of attention in the preparation of visually guided saccadic eye movements in man. In J. K. O'Regan \& A. Levey-Schoen (Eds.), Eye movements: From physiology to cognition (pp. 37-45). New York: North-Holland
McDowd, J. M., \& Oseas-Kreger, D. M. (1991). Aging, inhibitory processes, and negative priming. Journals of Gerontology, 46 , P340-P345. doi:10.1093/geronj/46.6.P340

Miller, E. K., Erickson, C. A., \& Desimone, R. (1996). Neural mechanisms of visual working memory in prefrontal cortex of the macaque. The Journal of Neuroscience, 16, 5154-5167

Milner, B. (1964). Some effects of frontal lobectomy in man. In J. M. Warren \& K. Akert (Eds.), The frontal granular cortex and behavior (pp. 313-334). New York: McGraw-Hill

Moores, E., Latti, L., \& Chelazzi, L. (2003). Associative knowledge controls deployment of visual selective attention. Nature Neuroscience, 6, 182-189. doi:10.1038/nn996

Moran, J., \& Desimone, R. (1985). Selective attention gates visual processing in the extrastriate cortex. Science, 229, 7782-7784. doi:10.1126/science. 4023713

Olivers, C. N. L. (2009). What drives memory-driven attentional capture: The effects of memory type, display type, and search type. Journal of Experimental Psychology: Human Perception and Performance, 35, 1275-1291. doi:10.1037/a0013896

Olivers, C. N. L., Meijer, F., \& Theeuwes, J. (2006). Feature-based memory-driven attentional capture: Visual working memory content affects visual attention. Journal of Experimental Psychology: Human Perception and Performance, 32, 1243-1265. doi:10.1037/0096-1523.32.5.1243

Palmer, S. E. (1999). Vision science: Photons to phenomenology. Cambridge, MA: MIT Press

Pashler, H., \& Shui, L.-P. (1999). Do images involuntarily trigger search? A test of Pillsbury's hypothesis. Psychonomic Bulletin \& Review, 6, 445-448

Paulesu, E., Frith, C. D., \& Frackowiak, R. S. J. (1993). The neural correlates of the verbal component of working memory. Nature, 362, 342-344. doi:10.1038/362342a0

Posner, M. I., \& Peterson, S. E. (1990). The attention system of the human brain. Annual Review of Neuroscience, 13, 25-42. doi:10.1146/annurev.ne.13.030190.000325

Postle, B. R. (2006). Working memory as an emergent property of the mind and brain. Neuroscience, 139, 23-38. doi:10.1016/j. neuroscience.2005.06.005

Rainer, G., Asaad, W. F., \& Miller, E. K. (1998). Selective representation of relevant information by neurons in the primate prefrontal cortex. Nature, 393, 577-579. doi:10.1038/31235

Reeves, A., \& Sperling, G. (1986). Attention gating in short-term visual memory. Psychological Review, 93, 180-206. doi:10.1037/ 0033-295X.93.2.180

Roelfsema, P. R., Lamme, V. A. F., \& Spekreijse, H. (1998). Objectbased attention in the primary visual cortex of the macaque monkey. Nature, 395, 376-381. doi:10.1038/26475

Rypma, B., \& D'Esposito, M. (1999). The roles of prefrontal brain regions in components of working memory: Effects of memory load and individual differences. Proceedings of the National Academy of Sciences, 96, 6558-6563. doi:10.1073/pnas.96.11.6558

Schneider, W., Eschman, A., \& Zuccolotto, A. (2002). E-Prime user's guide. Pittsburgh: Psychology Software Tools, Inc.

Schumacher, E. H., Lauber, E., Awh, E., Jonides, J., Smith, E. E., \& Koeppe, R. A. (1996). PET evidence for an amodal verbal working memory system. Neuroimage, 3, 79-88. doi:10.1006/ nimg.1996.0009

Shomstein, S., \& Yantis, S. (2002). Object-based attention: Sensory modulation or priority setting. Perception \& Psychophysics, 64, $41-51$

Smith, E. E., Jonides, J., Koeppe, R. A., Awh, E., Schumacher, E. H., \& Minoshima, S. (1995). Spatial versus object working memory: FET investigations. Journal of Cognitive Neuroscience, 7, 337 356. doi:10.1162/jocn.1995.7.3.337

Smyth, M. M. (1996). Interference with rehearsal in spatial working memory in the absence of eye movements. The Quarterly 
Journal of Experimental Psychology, 49A, 940-949. doi:10. 1080/027249896392379

Soto, D., Heinke, D., Humphreys, G. W., \& Blanco, M. J. (2005). Early, involuntary top-down guidance of attention from working memory. Journal of Experimental Psychology: Human Perception and Performance, 31, 248-261. doi:10.1037/0096-1523.31.2.248

Soto, D., \& Humphreys, G. W. (2007). Automatic guidance of visual attention from verbal working memory. Journal of Experimental Psychology: Human Perception and Performance, 33, 730-757. doi:10.1037/0096-1523.33.3.730

Soto, D., \& Humphreys, G. W. (2008). Stressing the mind: The effect of cognitive load and articulatory suppression on attentional guidance from working memory. Perception \& Psychophysics, 70, 924-934. doi:10.3758/PP.70.5.924

Soto, D., Humphreys, G. W., \& Heinke, D. (2006). Dividing the mind: The necessary role of the frontal lobes in separating memory from search. Neuropsychologia, 44, 1282-1289. doi:10.1016/j. neuropsychologia.2006.01.029

Stolz, J. A. (1996). Exogenous orienting does not reflect an encapsulated set of processes. Journal of Experimental Psychology: Human Perception and Performance, 22, 187-201. doi:10.1037/0096-1523.22.1.187
Stroop, J. R. (1935). Studies of interference in serial verbal reactions. Journal of Experimental Psychology, 18, 643-662.

Supèr, H., Spekreijse, H., \& Lamme, V. A. F. (2001). A neural correlate of working memory in the monkey primary visual cortex. Science, 293, 120-124. doi:10.1126/science.1060496

Theeuwes, J. (1992). Perceptual selectivity for color and form. Perception \& Psychophysics, 51, 599-606

Vogt, J., De Houwer, J., Moors, A., Van Damme, S., \& Crombez, G. (2010). The automatic orienting of attention to goal-relevant stimuli. Acta Psychologica, 61-69. doi:10.1016/j.actpsy.2009.12.006

Wilson, F. A. W., Scalaidhe, S. P. O., \& Goldman-Rakic, P. S. (1993). Dissociation of object and spatial processing domains in primate prefrontal cortex. Science, 260, 1955-1958. doi:10.1126/ science. 8316836

Wojciulik, E., \& Kanwisher, N. (1999). The generality of parietal involvement in visual attention. Neuron, 23, 747-764. doi:10.1016/S0896-6273(01)80033-7

Woodman, G. F., \& Luck, S. J. (2007). Do the contents of visual working memory automatically influence attentional selection during visual search? Journal of Experimental Psychology: Human Perception and Performance, 33, 363-377. doi:10. 1037/0096-1523.33.2.363 